\title{
Scolicidal activity of taurolidine for the treatment of hydatid disease
}

\author{
Aydin $\mathrm{I}^{1}$, Teksoz $\mathrm{S}^{1}$, Aytac $\mathrm{E}^{1}$, Polat $\mathrm{E}^{2}$, Ramazanoglu $\mathrm{R}^{3}$, Ozcan $\mathrm{M}^{1}$ \\ Department of General Surgery, Istanbul University, Cerrahpasa Medical Faculty, Istanbul, Turkey.
} serkanteksoz@gmail.com

\begin{abstract}
Objectives: In this experimental study, we have evaluated in vivo and in vitro activities of taurolidine (TRD) against protoscolices of Echinococcus granulosus.

Background: Scolicidal agent application is mandatory for the interventional treatment of hydatid cysts. Serious adverse effects of current scolicidal agents forced the scientist to search for less toxic chemicals.

Methods: One milliliter of protoscolices suspension was administered into twelve Petri's dishes, six of them containing $5 \mathrm{ml}$ of isotonic saline, and six of them containing $5 \mathrm{ml}(5 \mathrm{mg} / \mathrm{ml}) \mathrm{TRD}$. Forty, male Balb/c mice were divided into five equal groups. Group 1: protoscolices inoculated group; Group 2: protoscolices inoculated and thereafter immediately administered with a single dose intravenous (IV) $0.5 \mathrm{ml}$ TRD (400 mg/kg); Group 3: protoscolices inoculated and thereafter immediately administered with a single dose intraperitoneal (IP) $0.5 \mathrm{ml}$ TRD (400 mg/kg); Group 4: three months after inoculating the protoscolices, one day before the sacrifice single dose IV $0.5 \mathrm{ml}$ TRD (400 mg/kg) administered; Group 5: three months after inoculating the protoscolices, one day before the sacrifice single dose IP $0.5 \mathrm{ml}$ TRD $(400 \mathrm{mg} / \mathrm{kg})$ administered.

Results: All the protoscolices were dead in TRD-added Petri's Dishes in 90 minutes. In group 2 and group 3, no mouse had an intraabdominal hydatid cyst.

Conclusion: The results of this study are encouraging us to suggest TRD as an alternative scolicidal agent. Further clinical studies are needed to define the effectiveness and the mode of application of TRD for the treatment of hydatid disease (Tab. 1, Fig. 4, Ref. 30). Full Text in PDF www.elis.sk.

Key words: taurolidine, hydatid disease, scolicidal agent, Echinococcus granulosus.
\end{abstract}

Hydatid disease (HD) is a zoonosis caused by larvae of Echinococcus granulosus which form cysts in different organs of human and herbivores. Despite its benign nature, Echinococcus granulosus causes serious financial and manpower loss and even death in case of complications (1). Medical treatment, percutaneous drainage and surgical treatment are current treatment options of intra abdominal $\operatorname{HD}(2,3)$. Use of scolicidal agents is a mandatory step in the interventional treatment of hydatid cysts. By killing scolices, scolicidal agents assure the disinfection of the cyst and prevent contamination of the surgical field. Hypertonic sodium chloride, silver nitrate, povidone iodine, cetrimide are frequently used scolicidal agents. However, these scolicidal agents could cause acute or chronic toxicity $(4,5)$. An ideal scolicidal agent should be highly effective in low concentrations and in short time, should not have local or systemic side-effects, should not harm liver or bile ducts, should have low viscosity, should be prepared and conserved easily and be inexpensive (4-6). But there is no agent that fulfills all of these specifications.

Taurolidine (TRD), bis-( 1,1-dioxo-perhydro-1,2,4-thiadiazinyl-4)-methan, consists of two taurinamide rings derived from the naturally occurring amino sulfonic acid, taurine and three mole-

${ }^{1}$ Department of General Surgery, Istanbul University, Cerrahpasa Medical Faculty, Istanbul, Turkey, ${ }^{2}$ Department of Microbiology, Istanbul University, Cerrahpasa Medical Faculty, Istanbul, Turkey, and ${ }^{3}$ Department of Pathology, Istanbul University, Cerrahpasa Medical Faculty, Istanbul, Turkey Address for correspondence: Serkan Teksoz, MD, Cerrahpasa Tip Fakultesi, Genel Cerrahi AD. Kat: 3, 34098 Fatih, Istanbul, Turkey. Phone: +902124143000-23002 cules of formaldehyde combining to form a two-ringed structure bridged by a methylene group. Intravenous (IV) five-day TRD treatment showed beneficial effects in septic patients without causing any side effects (7). TRD has also been used intraperitoneally for the treatment of peritonitis (8). In this experimental study, we have evaluated the in vivo and in vitro activities of TRD against protoscolices of Echinococcus granulosus.

\section{Materials}

This study was performed in the Experimental Research Laboratory of Istanbul University Cerrahpasa Medical Faculty after approval of the Istanbul University Experimental Medicine Research Institute Ethical Committee (Protocol No: 47/2008).

\section{Preparation of protoscolices}

The live protoscolices were harvested intraoperatively from liver hydatic cysts (Echinococcus granulosus) of patients by suction of the virgin cyst fluid. Infected or bile-stained aspirates were discarded. Submerged protoscolices were collected, washed with isotonic saline and decanted (not centrifuged) for three times to get a purifed scolices suspension. Thus, a suspension of 1,000-1,200 scolices/ml was obtained. Then, the viability of scolices was evaluated based on their morphology, motility and vital dye (eosin) uptake.

In vitro study

One milliliter of this suspension was put in twelve Petri's 


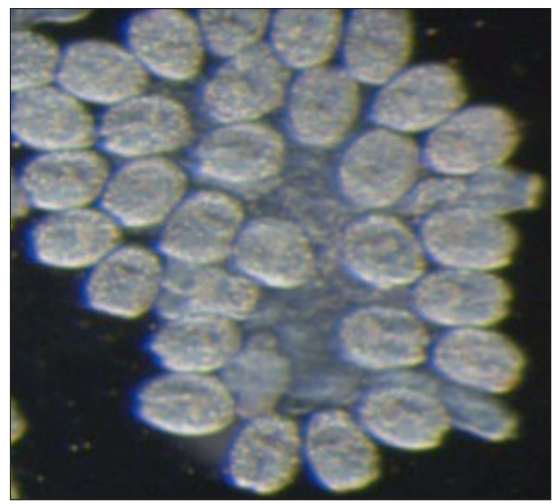

Fig. 1. Alive protoscolex.

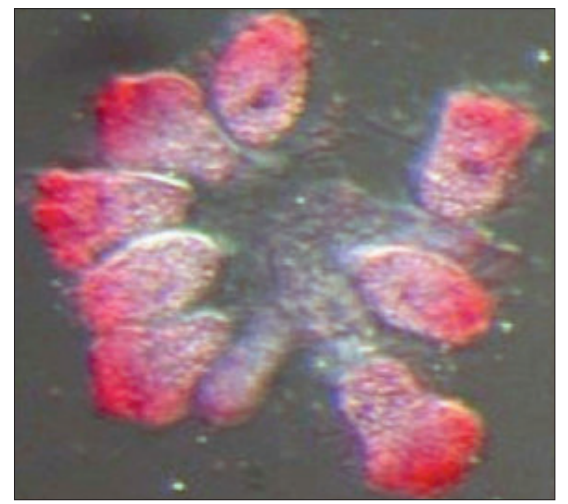

Fig. 2. Dead protoscolex.

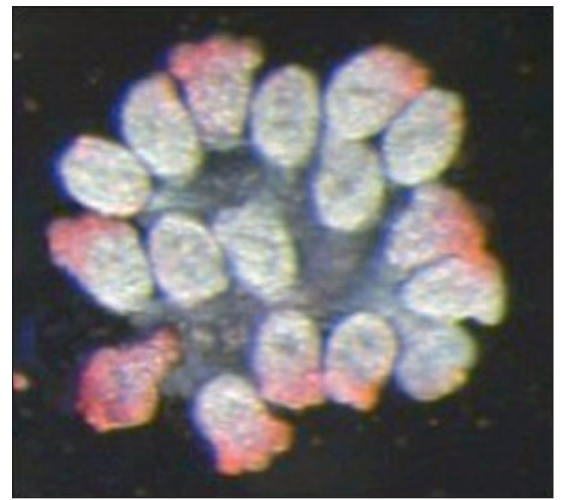

Fig. 3. Appearance of protoscolex at 90 th $\mathrm{mi}-$ nute after taurolidine application.
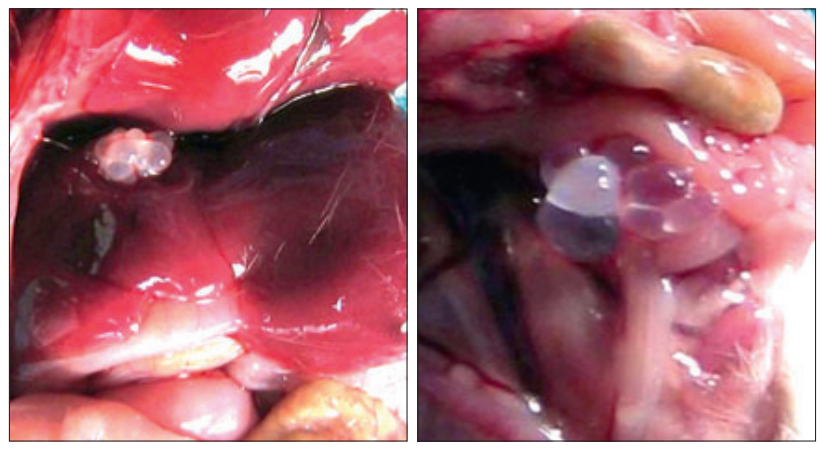

Fig. 4. Intraabdominal hydatid cyst.

dishs, six of them containing $5 \mathrm{ml}$ of isotonic saline, and six of them containing $5 \mathrm{ml}(5 \mathrm{mg} / \mathrm{ml})$ TRD. One drop of $0.1 \%$ eosin was added to each Petri's dish to confirm or disprove the death of protoscolices. Ten different fields of each Petri's dish were examined under 50x and 100x magnifications with a light microscope. The viabilities were calculated as percentage by counting live protoscolices in the microscope area. Protoscolices that conserved their ovoid shape, did not lose their ameboid movements and did not stain with eosin were assumed alive. The ones that were immotile, stained with eosin and whose rostella were retracted, thus becoming spherical were considered dead (Fig. 1-4).

In vivo study

After showing scolicidal activity of TRD in vitro, we designed an in vivo experiment. Forty young (eight-week old), male, Balb/c

Tab. 1. The experimental groups of the in vivo study.

\begin{tabular}{lcccc}
\hline & $\begin{array}{c}\text { IP } \\
\text { protoscelex } \\
\text { inoculation }\end{array}$ & $\begin{array}{c}\text { TRD } \\
\text { administration }\end{array}$ & $\begin{array}{c}\text { Application } \\
\text { route of } \\
\text { TRD }\end{array}$ & TRD dose \\
\hline Group 1 & + & - & - & - \\
Group 2 & + & + & I.V $*$ & $0.5 \mathrm{cc}(400 \mathrm{mg} / \mathrm{kg})$ \\
Group 3 & + & + & I.P* & $0.5 \mathrm{cc}(400 \mathrm{mg} / \mathrm{kg})$ \\
Group 4 & + & + & I.V $* *$ & $0.5 \mathrm{cc}(400 \mathrm{mg} / \mathrm{kg})$ \\
Group 5 & + & + & I.P $* *$ & $0.5 \mathrm{cc}(400 \mathrm{mg} / \mathrm{kg})$ \\
\hline
\end{tabular}

* - Taurolidine administration at the mean time with protoscelex inoculation, ** Taurolidine administration three months after protoscelex inoculation, IP - intraperitoneal, IV - intravenous, TRD - taurolidine mice of 24-30 g weight, were divided into five equal groups $(n=8$ for per group) (Tab. 1). The mice were looked after in accordance with the Guide for the Care and Use of Laboratory Animals (NIH Publication no. 86-23, revised 1985), maintained in colony cages under controlled light, temperature and humidity conditions and permitted ad libitum access to standard lab chow and tap water during the experimental procedures. Protoscolices were inoculated intraperitoneally under aseptic conditions. Group 1: protoscolices inoculated group; Group 2: protoscolices inoculated and immediately thereafter administered with a single dose IV $0.5 \mathrm{ml} \mathrm{TRD}$ (400 mg/kg); Group 3: protoscolices inoculated and immediately thereafter administered with a single dose IP $0.5 \mathrm{ml}$ TRD (400 mg/ $\mathrm{kg}$ ); Group 4; three months after inoculating the protoscolices, one day before the sacrifice a single dose IV $0.5 \mathrm{ml}$ TRD (400 mg/kg) administered; Group 5: three months after inoculating the protoscolices, one day before the sacrifice a single dose IP 0.5 $\mathrm{ml}$ TRD $(400 \mathrm{mg} / \mathrm{kg})$ administered. The mice were sacrificed 90 days after the protoscolices inoculation. Intraperitoneal hydatid cyst development and viability of cyst content were evaluated.

\section{Statistical analysis}

Kruskal-Wallis and post hoc Bonferoni adjusted Mann-Whitney tests were used for the statistical analysis, while $\mathrm{p}<0.01$ was considered significant.

\section{Results}

In our preliminary evaluation, we had observed that a single dose IV, IP and intrabiliary TRD (400 mg/kg) administration had caused no toxic effects upon the peritoneum, hepatocytes and cholangiocytes of the Balb/c mice.

In vitro, the movement of protoscolices stopped and the protoscolices got circular shape seven minutes after TRD application. Forty-five minutes after TRD administration, $50 \%$ of the protoscolices and ninety minutes after the administration all protoscolices were dead in TRD-added Petri's dishes (Fig. 3). However, at the end of the same period, $95 \%$ of protoscolicesesices were alive in saline group.

When we evaluate the in vivo study, no mouse died during the experiment. In group 1, seven mice (7/8) had intraabdominal hy- 
648-651

datid cysts which were located on the liver and intestines. These cysts contained live protoscolices. The mice in group $2(0 / 8)$ and group $3(0 / 8)$ had no intraabdominal hydatid cysts. These values for group 2 and group 3 are significantly lower than for group 1 $(p<0.001)$. Seven mice had intraabdominal hydatid cysts in each of groups 4 (7/8) and 5 (7/8) but none of the hydatid cysts contained alive protoscolices.

\section{Discussion}

HD is currently an important public health problem especially in the developing countries including Turkey. Many clinical and experimental studies have been published about the treatment of the disease; nevertheless none of them has defined an ideal scolicidal agent (9). Protoscolices cause recurrent hydatid cyts in the liver. Inoculation of protoscolices into the peritoneal cavity of a young mouse is an experimental model of hydatidosis $(10,11)$.

Serious adverse effects of current scolicidal agents forced the physicians to search for less toxic chemicals to be used for this purpose. Formalin is one of the former drugs but it has serious systemic toxic effects and causes epithelial proliferation in biliary tract so it is not currently used in clinical practice (12). Ethyl alcohol at $98 \%$ concentration is used for percutaneous treatment of hepatic hydatid cyst. Its effectiveness depends on concentration of alcohol while sclerosing cholangitis could occur after ethyl alcohol administration (13). Additionally, ethyl alcohol is not preferred during the surgical treatment of the hydatid cysts since it is flammable and volatile. Hypertonic sodium chloride and silver nitrate are used as scolicidal drug but they could cause acute pancreatitis and sclerosing cholangitis $(4,14)$. Hypernatremia is a foreseeable complication of hypertonic sodium treatment (15). Cetrimide-chlorhexidine combination has an adhesive action on small bowel (16). Hypertonic sodium chloride and alcohol inactivate and degenerate the germinative membrane. Alcohol also obliterates residual cavity beside its scolicidal activity (15). Scolicidal effect of povidoneiodine which is used mostly in our country depends on dosage, it can also protect secondary ecinococcosis $(4,17)$. Allergic reactions could be seen after application of povidone-iodine. Considering these adverse effects of the current scolicidal agents, the discovery of less toxic and much effective chemicals are urgently needed.

The scolicidal effects of TRD are under investigation. Ekci et al (18) suggested that TRD has no scolicidal activity. However, the design of the methodology was not compatible with the clinical scenario in this pilot study. Our in vitro application time for TRD was longer and we administed TRD intraabdominally after inoculating the protoscolices into the abdomen. They had inoculated TRD-treated protoscolices into the abdominal cavity and did not evaluate the exact application time (18). In the current experimental study, in vitro and in vivo scolicidal activities of TRD have been shown. TRD has a therapeutic effect against many infections causing peritonitis when administered at the doses that we used in the current study. Additionally, no serious adverse effects were reported after administration of TRD infusion in human studies (19). TRD is presented as an antibacterial and antineoplastic agent in literature. Active metabolites of TRD, taurul- tam and taurinamide, interact with the structures of bacterial wall (20-23). Antioxidant effects of TRD could attenuate the immune response against the parasitic infections (24). TRD has beneficial effects on wound healing process too $(25,26)$. TRD could support physiologic mechanisms by reducing the harmful effects of the pathology. Parasitic infections could be harmful via producing endotoxins. TRD also neutralizes bacterial endotoxins, exotoxins, and lipopolysaccharides (27-29). TRD has anti-adherent and antiinflammatory activities (7-8). One or more of these characteristics could be responsible for the scolicidal activity of TRD.

Formaldehyde is one of the degradation products of TRD. Carcinogenetic and cytotoxic effects of formaldehyde are known. However, the drug (Taurolin ${ }^{\circledR}$, Boehringer Ingelheim, Germany) that we used in the study includes a stabilizer, polyvinylpyrrolidone, for reducing formaldehyde concentration (30). No cancer development and no carcinogenetic pathways were reported to be related to TRD treatment.

The results of this study are encouraging us to suggest TRD as an alternative scolicidal agent. Reaching the maximum scolicidal activity could take a longer time for TRD but it could be less toxic than other scolicidal agents. Further clinical studies are needed to define the effectiveness and the mode of application of TRD for the treatment of hydatid disease.

\section{References}

1. Goksoy E, Duren M. Operative therapie des Echinococcus granulosus (cysticus). Chirurg 2000; 71:21-29.

2. Kapan M, Yavuz N, Kapan S, Polat S, Goksoy E. Totally laparoscopic pericystectomy in hepatic hydatid disease. J Laparoendosc Adv Surg Tech A 2004; 14: 107-109.

3. Goksoy E, Saklak M, Saribeyoglu K, Schumpelick V. Chirurgische Therapie bei Echinococus-Zysten der Leber. Chirurg 2008; 79: 729-737.

4. Karayalcin K, Aras N. Use of scolicidal agents in hydatid cyst surgery. T Klin J Surgery 1998; 3: 216-218.

5. Eyupoglu B, Doganay M, Reis E, Yuksek YN, Kulacoglu S, Kama NI. The effects of scolocidal agents on hepatopancreaticobiliary system “An experimenal study”. Turk J Gastroenterol 1999; 10: 280-286.

6. Smego RA Jr, Sebanego P. Treatment options for hepatic cystic echinococcosis. Int J Infect Dis 2005; 9: 69-76.

7. Willatts S.M, Radford S, Leitermann M. Effect of the antiendotoxic agent, taurolidine, in the treatment of sepsis syndrome: A placebo-controlled, double-blind trial. Crit Care Med 1995; 23: 1033-1039.

8. Schneider A, Sack U, Rothe K, Bennek J. Peritoneal taurolidine lavage in children with localised peritonitis due to appendicitis. Pediatr Surg Int 2005; $21: 445-448$.

9. Mottaghian H, Saidi F. Postoperative recurrence of hydatid disease. Br J Surg 1978; 65: 237-242.

10. Urrea-Paris MA, Casado N, Moreno MJ Rodriguez-Caabeiro F. Chemoprophylactic praziquantel treatment in experimental hydatidosis. Parasitol Res 2001; 87: 510-512.

11. Puryan K, Karadayi K, Topcu O, Canbay E, Sumer Z, Turan M, Karayalcin K, Sen M. Chlorhexidine gluconate: an ideal scolicidal agent in the treatment of intraperitoneal hydatidosis? World J Surg 2005; 29: 227-230. 
12. Houry S, Bedossa P, Languille O, Huguier M, Lemaigre G. Experimental sclerosing cholangitis following intrabiliary injection of formol in the rat. Chirurgie 1992; 118: 683-686.

13. Castellano G, Moreno-Sanchez D, Gutierrez J, Moreno-Gonzalez E, Colina F, Solis-Herruzo JA. Caustic sclerosing cholangitis. Report of four cases and a cumulative review of the literature. Hepatogastroenterology 1994; 41: 458-470.

14. Belghiti J, Benhamou J.P, Houry S, Grenier P, Huguier M, Fékété F. Caustic sclerosing cholangitis. Arch Surg 1986; 121: 1162-1165.

15. Sahin M, Eryilmaz R, Bulbuloglu E. The effect of scolicidal agents on liver and biliary tree (experimental study). J Invest Surg 2004; 17: 323-326.

16. Topcu O, Kuzu I, Karayalcin K. Effects of peritoneal lavage with scolicidal agents on survival and adhesion formation in rats. World J Surg 2006; 30: 127-133.

17. Gokce O, Gokce C, Yılmaz M, Huseyinoglu K, Gunel S. Povidoneiodine in experimental hydatidosis. Br J Surg 1991; 78: 495-496.

18. Ekci B, Gurol Y, Aydin I, Yalniz F, Ozcan M, Zengin K. The protoscolicidal effect of $1 \%$ polyvinylpyrrolidone- iodine (Pvp-1) and 2\% taurolidine on abdominal hydatidosis. Turkiye Parazitol Derg 2010; 34: 152-155.

19. Gong L, Greenberg HE, Perhach JL, Waldman SA, Kraft WK. The Pharmacokinetics of taurolidine metabolites in healthy volunteers. J Clin Pharmacol 2007; 47; 697-703.

20. Browne MK. Pharmacological and clinical studies on Taurolin. In: Bruckner WL, Pfirrmann RW (Eds). A New Concept in Antimicrobial Chemotherapy for Surgical Infection, Baltimore: Urban \& Schwarzenberg, 1985, $51 \mathrm{pp}$.

21. Browne MK. The treatment of peritonitis by an antiseptic - Taurolin. Pharmatherapeutica 1981; 2: 517-522.
22. Blenkharn JJ. Sustained anti-adherence activity of taurolidine (Taurolin) and noxythiolin (Noxyflex S). J Pharmacol 1988; 40: 509-511.

23. Gorman SP, McCafferty DF, Woolfson AD, Jones DS. Electron and light microscope observations of bacterial cell surface effects due to taurolidine treatment. Lett Appl Microbiol 1987; 4: 103-109.

24. Konukoglu D, Iynem H, Ziylan E. Antioxidant status in experimental peritonitis: effects of alpha tocoferol and Taurolin. Pharmacol Res 1999; 39: $247-251$.

25. Jiborn H, Ahonen J, Zederfeldt B. Healing of experimental colonic anastomosis collagen metabolism in the colon after left colon resection. Am J Surg 1980; 139: 398-405.

26. Akkuş A, Gülmen M, Cevik A, Bildik N, Sad O, Oztürk E, Barişik NO. Effect of peritoneal lavage with taurolidine on primary colonic anastomosis in a rat model of secondary peritonitis. Surg Today 2006; 36 : 436-440.

27. Monson JR, Ramsey PS, Donohue JH. Taurolidine inhibits tumor necrosis factor (TNF) toxicity: new evidence of TNF and endotoxin synergy. Eur J Surg Oncol 1993; 3: 226-231.

28. Watson RW, Redmond HP, McCarthy J, Bouchier-Hayes D. Taurolidine, an antilipopolysaccharide agent, has immunoregulatory properties that are mediated by amino acid taurine. J Leukoc Biol 1995; 58: 299-306.

29. Leithauser ML, Rob PM, Sack K. Pentoxifylline, cyclosporine A and taurolidine inhibit endotoxin-stimulated tumor necrosis factor-alpha production in rat mesangial cell cultures. Exp Nephrol 1997; 1: 100-104.

30. Kirsch LE, Sihn YS. The effect of polyvinylpyrrolidine on the stability of taurolidine. Pharm Dev Technol 1997; 2: 345-356.

Received July 12, 2011. Accepted January 17, 2012. 